\title{
FONCTIONNEMENT D'UNE POPULATION D'ÉPERLAN (OSMERUS EPERLANUS, OSMÉRIFORMES OSMERIDAE) SITUÉE EN LIMITE MÉRIDIONALE DE SON AIRE DE RÉPARTITION, INFLUENCE DE LA TEMPÉRATURE.
}

\author{
O. PRONIER (1) et E. ROCHARD (2)
}

(1) Cemagref, Unité Ressources Ichthyologiques en plans d'eau, 361 rue J.F. Breton, BP 5095 Montpellier Cedex 01, France.

(2) Cemagref, Unité Ressources Aquatiques Continentales, Equipe poissons migrateurs et pêche continentale, 50 avenue de Verdun, 33612 Cestas Cedex, France.

\section{RÉSUMÉ}

Ce travail présente le fonctionnement de la population d'éperlan Osmerus eperlanus dans le bassin versant de la Gironde entre 1979 et 1996 et examine les liens possibles avec la température.

Les données utilisées proviennent d'un suivi de l'ichtyofaune effectué mensuellement sur un transect situé à $43 \mathrm{~km}$ de l'embouchure de la Gironde (zone oligohaline) dans le cadre du suivi lié à l'installation de la centrale nucléaire de Braud et St-Louis. L'échantillonnage effectué sur cinq stations de surface et sur cinq stations de fond a permis une estimation de la densité de la population.

Ce petit migrateur amphihalin, situé en limite méridionale de son aire de répartition, a présenté durant la période d'étude une très forte diminution de sa fréquence et de son abondance alors que les impacts anthropiques le concernant ne semblent pas avoir augmenté. Durant la même période, la température de l'eau de l'estuaire mesurée dans la même zone et corrigée des variations saisonnières s'est élevée $\left(+2{ }^{\circ} \mathrm{C}\right)$. Nous avons examiné le lien éventuel entre ces deux phénomènes concomitants et analysé les répercussions éventuelles de la température sur la dynamique de cette population.

Pour cela, nous avons établi une première relation stock/recrutement entre les effectifs de géniteurs et ceux des juvéniles en provenant, relation qui a été confrontée aux températures.

Nous avons également procédé à une analyse des taux de mortalité instantanés par rapport aux températures moyennes correspondantes. On constate que la mortalité augmente avec la température.

Outre son action sur la mortalité, nous posons l'hypothèse d'une action indirecte de la température qui, en ralentissant la croissance, ferait passer l'âge de première maturité sexuelle de 1 an à 2 ans.

Le cycle de vie relativement court de l'éperlan et donc sa rapidité de réaction aux variations environnementales pourrait en faire un modèle intéressant pour l'étude du fonctionnement des populations de salmoniformes. 
Mots-clés : Osmerus eperlanus, dynamique de population, écologie, température, estuaire.

\section{WORKING OF A SMELT (OSMERUS EPERLANUS, OSMERIFORMS OSMERIDAE) POPULATION LOCATED AT THE SOUTH LIMIT OF THE SPECIES DISTRIBUTION AREA, INFLUENCE OF THE TEMPERATURE.}

\section{ABSTRACT}

This paper investigates the involvement of temperature in the dynamics of the smelt Osmerus eperlanus population in the Gironde basin between 1979 and 1996.

Data come from a monthly ichtyologic follow-up on Braud St-Louis nuclear power station, $43 \mathrm{~km}$ away from the mouth of the Gironde (oligohaline zone). The density of population was estimated through a sampling carried out at 5 surface stations and 5 deep stations. Located in the south of the species' distribution area, this population has shown during the studied period a drastic decrease in its frequency and abundance level whereas anthropogenic impacts did not seem to change. During the same period and in the same area, the water temperature in the estuary increased by $2{ }^{\circ} \mathrm{C}$. As a result, we analysed the probable link between those 2 phenomena and the possible impact of this modification on the evolution of the population dynamics. For that purpose, we have established a stock/recruitment relationship between broodstock and induced fry and have linked it to the temperature values. We have also analysed the relationship of instantaneous mortality rate with temperatures. We have noticed that high values of mortality rates occur while temperatures are the highest. Moreover, temperature seems to slow down the smelt's growth in the Gironde basin.

The smelt's life cycle being relatively short, this fish reacts strongly and quickly to the environmental fluctuations. Thus, it is an interesting model for studying further salmoniforms population dynamics.

Key-words : Osmerus eperlanus, population dynamics, ecology, temperature, estuary.

\section{INTRODUCTION}

Cet article a pour objectif de mettre en évidence l'évolution sur le moyen terme de la population d'éperlan Osmerus eperlanus L. (Osmériformes, Osmeridae) de l'estuaire de la Gironde et d'examiner une éventuelle relation avec la température de l'eau. Pour cela, nous avons choisi d'analyser les fluctuations de quelques caractéristiques de la dynamique de cette population (taux de mortalité, relation stock-recrutement) en fonction de la variation de la température.

La température agit sur l'activité biologique des êtres vivants selon des relations de plusieurs types (LISCHKE et al., 1997) qui peuvent être résumées par la loi de tolérance de SHELFORD (GIBBONS, 1976 ; RAMADE, 1987). On définit ainsi théoriquement une zone optimum encadrée par deux zones de tolérance, le tout borné par deux zones létales. A court terme, il est relativement aisé, expérimentalement, de définir les limites de tolérance des individus, que ce soit à la chaleur (COX, 1974 ; MAC FARLANE et al., 1976) ou au froid (COX et COUTANT, 1976). Par contre, il est nettement plus difficile de déterminer ces 
limites sur le moyen terme au niveau d'une population (succession de stades écologiques avec des exigences différentes et hétérogénéité thermique de l'aire de distribution et des habitats utilisés) (HUTCHISON, 1976). Plus rares que ceux basés sur l'étude de la physiologie des animaux à différentes températures, les travaux traitant de l'influence de ce facteur sur les populations ont connu deux vagues successives d'intérêt. La première, liée au développement des centrales thermiques puis nucléaires (GIBBONS et SHARITZ, 1974 ; ESCH et MAC FARLANE, 1976), la seconde aux travaux sur le réchauffement global et l'effet de serre (KAREIVA et al., 1993 In KELEHER et RAHEL, 1996 ; WOOD et MAC DONALD, 1997). Dans le cadre des travaux actuels sur les modifications climatiques et leurs conséquences écologiques à l'échelle des populations, il a été montré que l'augmentation de la température peut entraîner de fortes modifications dans la localisation, la forme et la taille des aires de répartition, que ce soit chez les végétaux (SCHENK, 1996) ou chez les animaux (KELEHER et RAHEL, 1996 ; EATON et SCHELLER, 1996). Chez les poissons d'eau froide, le réchauffement entraîne des pertes d'habitats et peut conduire au déplacement de populations (MAC CARTHY et HOULIHAN, 1997), à leur fragmentation, voire à leur disparition (KELEHER et RAHEL, 1996 ; RAHEL et al., 1996). Au niveau des populations on examine généralement en première approche, dans la zone la plus froide de l'aire de répartition, la température minimale supportable en hiver par l'espèce et dans la zone la plus chaude de l'aire de répartition, la température maximale supportable en été (KELEHER et RAHEL, 1996 ; RAHEL et al., 1996).

L'éperlan est une petite espèce d'origine paléartique (MAC ALLISTER, 1963) dont l'estuaire de la Gironde marque la limite méridionale de l'aire de répartition, la population qui $y$ vit se situe donc dans la zone de tolérance supérieure de sa niche thermique. Par ailleurs, plusieurs auteurs ont montré la sensibilité de cette espèce à la température (BORCHARDT, 1988 ; SEPULVEDA, 1994 ; LYLE et MAITLAND, 1997). Dans ce cas, il suffit d'une faible augmentation de la température de l'eau pour perturber de façon importante le cycle biologique de l'espèce, voire la décimer. Quelques-unes des populations nordiques d'Osmerus eperlanus sont strictement dulçaquicoles mais à l'échelle de l'aire de répartition, la plupart sont amphihalines potamotoques (NELLBRING, 1989). Parmi ces dernières, les caractéristiques écologiques varient en fonction de la latitude. Ainsi, par exemple, la reproduction de l'éperlan nécessite une température assez fraîche, ceci explique que la période de reproduction se situe en mai-juin au nord de l'aire de répartition (ALTUKHOV et YERASTOVA, 1974), en mars-avril au coeur de l'aire de répartition (MAITLAND, 1990 ; SEPULVEDA, 1994 ; THIEL et al., 1995 ; LYLE et MAITLAND, 1997) et en février-mars dans les zones les plus méridionales (BOIGONTIER et MOUNIÉ, 1984 ; LARDEUX, 1986).

La population qui fréquente le bassin versant Gironde-Garonne-Dordogne a un cycle écologique de type amphihalin potamotoque. En novembre-décembre, les adultes quittent les zones marines littorales et se regroupent dans l'estuaire de la Gironde, près de la limite de salure des eaux (BOIGONTIER et MOUNIÉ, 1984) (Figure 1). En février-mars, ils vont se reproduire dans la partie basse de la Garonne ou de la Dordogne en limite de marée dynamique et repartent ensuite en mer. Les jeunes arrivent dans l'estuaire en juin-juillet, ils y effectuent une partie importante de leur croissance avant de rejoindre les survivants de l'année d'avant. Pour les populations vivant au coeur de l'aire de répartition, l'âge à la première maturité sexuelle est de 1 an, leur longévité est de 4 à 5 ans et leur taille maximale est de $307 \mathrm{~mm}$ pour une moyenne de $85 \mathrm{~mm}$ (MAITLAND, 1990). Par contre, sur la partie septentrionale l'âge à la première maturité sexuelle est de 2 ans (Lt. moyenne $=150 \mathrm{~mm}$ ). Les travaux effectués sur la partie méridionale (BOIGONTIER et MOUNIÉ, 1984 ; LARDEUX 1986) indiquent que l'âge à la première maturité sexuelle est de 1 an pour une taille Lt. de $100 \mathrm{~mm}$. 


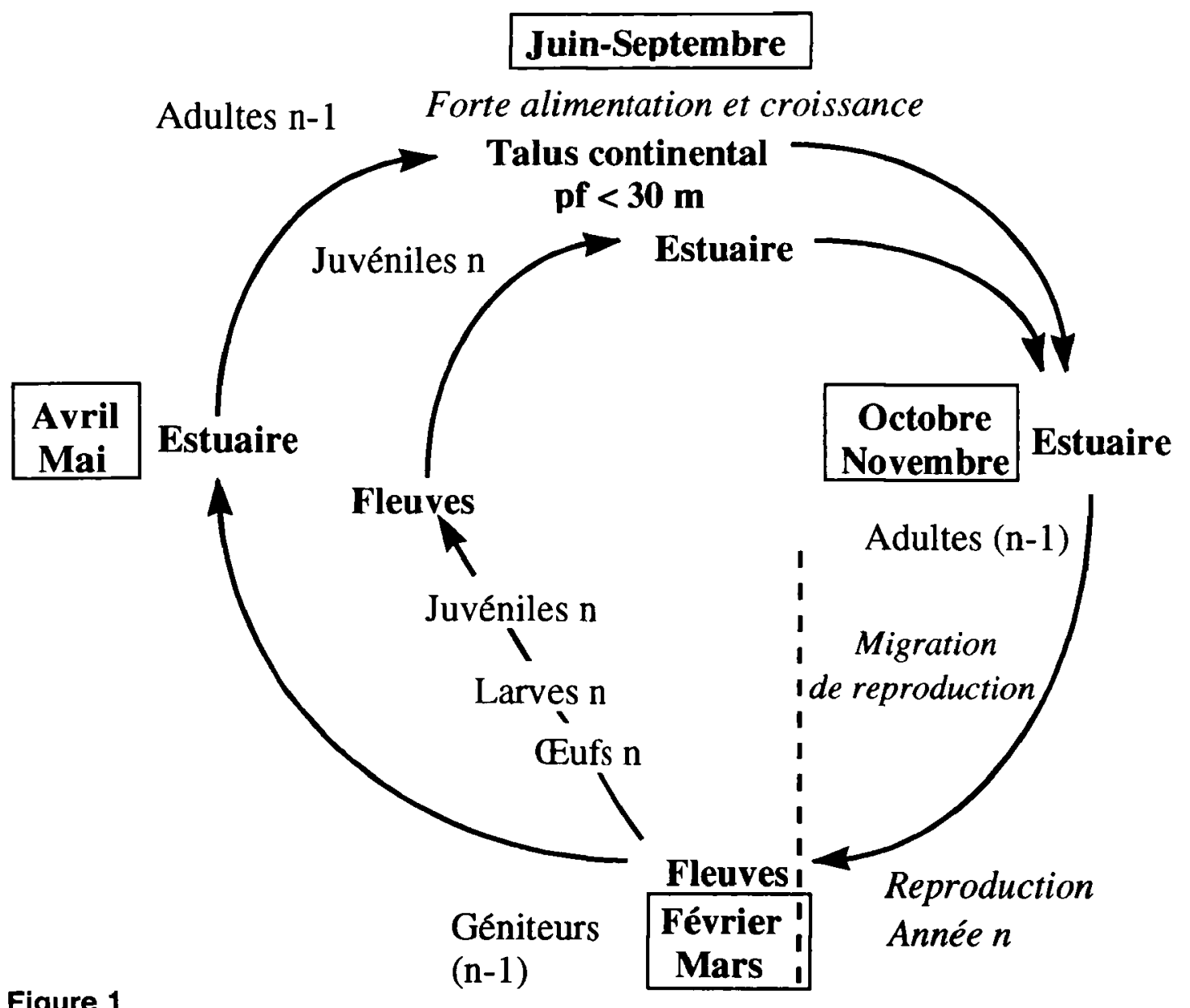

Figure 1

Schéma du cycle écologique d'une population amphihaline d'éperlan Osmerus eperlanus.

Figure 1

Life history of a diadromous population of smelt Osmerus eperlanus.

Outre les avancées qu'il permet de réaliser sur l'écologie de cette petite espèce d'intérêt halieutique et sur l'évolution de cette population sur le long terme, ce travail peut également contribuer à une meilleure compréhension de la dynamique d'une population de poisson migrateur située en limite géographique de sa zone de tolérance pour un facteur environnemental.

\section{MATÉRIEL ET MÉTHODES}

Les données utilisées ont été collectées par le Cemagref dans le cadre d'un suivi mensuel de l'ichtyofaune circulante de l'estuaire de la Gironde, lié à l'installation du Centre de Production Nucléaire du Blayais (ANONYME, 1979 ; CASTELNAUD et ROCHARD, 1996).

\section{Zone d'échantillonnage}

Les échantillons ont été prélevés entre avril 1979 et décembre 1996 sur 3 stations de surface et 3 stations de fond ( 5 stations de surface et 5 stations de fond, pour la période entre avril 1979 et janvier 1991) le long d'un transect situé dans la zone oligohaline, à $43 \mathrm{~km}$ de l'embouchure de l'estuaire de la Gironde (Figure 2). 


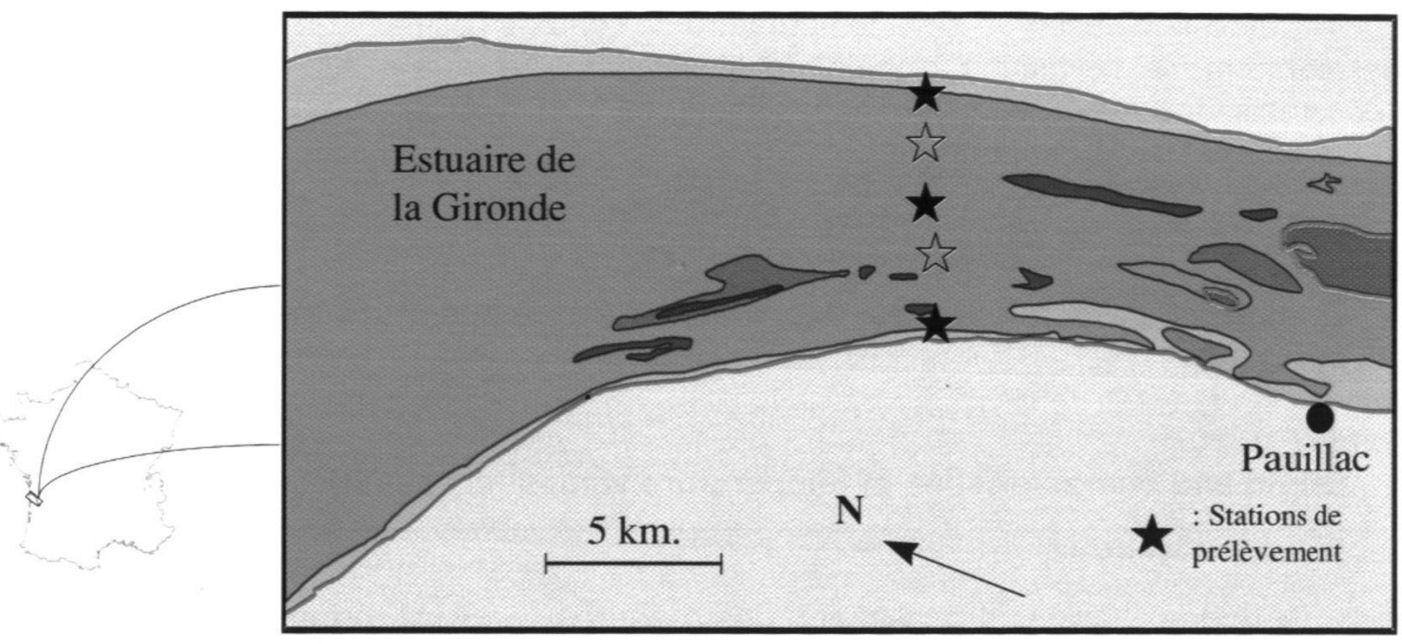

Figure 2

Situation géographique des stations de prélèvements dans l'estuaire de la Gironde. Les stations correspondant aux étoiles blanches n'ont été utilisées que d'avril 1979 à janvier 1991, les autres ont été échantillonnées sur toute la période.

\section{Figure 2}

Location of the sampling stations in the Gironde estuary. The white stars stations have been used only during April 1979-January 1991, the other stations have been sampled over the whole period.

Le protocole d'échantillonnage est identique à celui décrit par ELIE et ROCHARD (1994). Les prélèvements, d'une durée de 5 minutes, sont effectués à l'aide de filets poches, de jour, à contre-courant entre la moitié du flot et l'étale de pleine mer, à la fois en surface et au fond. Le navire se maintient face au courant avec une vitesse de 1,0 noeud par rapport au fond. En surface, nous avons utilisé 2 cadres rectangulaires $(4,0 \times 1,0 \mathrm{~m})$ portés de part et d'autre du bateau et, au fond, un traîneau suprabenthique $(2,0 \times 1,2 \mathrm{~m})$ maintenu à $0,2 \mathrm{~m}$ du sédiment par des patins.

Le maillage des deux filets est de $18 \mathrm{~mm}$ en maille étirée dans la partie principale de l'engin et de $1,4 \mathrm{~mm}$ dans la partie terminale. Des débitmètres (GENERAL OCEANICS 2030 R) placés à l'ouverture des engins permettent d'estimer le volume d'eau traversant les engins pendant la durée de chaque trait.

En nous basant sur des travaux réalisés sur la Loire (LARDEUX, 1986), nous avons considéré que les éperlans de la Gironde étaient adultes à partir de $100 \mathrm{~mm}$ (Lt.).

\section{Facteur thermique}

Ces mesures ont deux origines (Tableau I). Lors des prélèvements, elles ont été effectuées à l'aide d'une sonde multiparamètres YSI S.C.T. 33 sur des échantillons d'eau prélevés au fond ou en surface grâce à une bouteille de prélèvement à renversement. A la prise d'eau de la centrale nucléaire de Braud et St-Louis, la température est enregistrée à heure fixe à l'aide d'un thermographe.

L'action de la température a été envisagée sur le taux de mortalité de la population et sur la relation stock-recrutement. De façon à éliminer les variabilités saisonnières et à ne retenir qu'une tendance interannuelle, la série chronologique des données de température a été transformée en utilisant des moyennes mobiles pondérées sur 13 mois et 25 mois. 
L'évolution observée de la température de l'eau de l'estuaire de la Gironde (Figure 3) est conforme à l'évolution globale de la température sur le sud-ouest de la France, l'ensemble des suivis atmosphériques montrant une augmentation des niveaux de gaz carbonique et de la température.

\section{Tableau I}

Origine et caractéristiques des valeurs de température pour les différentes périodes.

\section{Table I}

Origin and characteristics of temperature values for the different periods.

\begin{tabular}{cccc}
\hline & Localisation des mesures & Fréquence & $\begin{array}{c}\text { Nombre } \\
\text { par jour }\end{array}$ \\
\hline 1979 à 1983 & $\begin{array}{c}\text { Sur les stations } \\
1983 \text { à } 1994\end{array}$ & $\begin{array}{c}\text { 3 jours par mois } \\
\text { A la prise d'eau de la centrale }\end{array}$ & 6 \\
1994 à 1995 & $\begin{array}{c}\text { Quotéaire du Blayais } \\
\text { Sur les stations }\end{array}$ & 3 jours par mois & 6 \\
\hline
\end{tabular}

De façon plus qualitative, les différentes années ont été groupées en quatre types en fonction de la rigueur relative de leur hiver (standard si $\mathrm{T}^{\circ} \mathrm{min} .>5^{\circ} \mathrm{C}$ et $<10^{\circ} \mathrm{C}$, froid si $\mathrm{T}^{\circ} \min .<5^{\circ} \mathrm{C}$ ) et de leur été (standard si $\mathrm{T}^{\circ} \max .>20^{\circ} \mathrm{C}$ et $<25^{\circ} \mathrm{C}$, chaud si $\mathrm{T}^{\circ}$ min. $>25^{\circ} \mathrm{C}$ ).

Nous avons également considéré pour les différentes périodes clés de l'espèce (abondance maximale de chacune des écophases) les températures minimale, maximale et moyenne.

\section{Calcul des fréquences}

Nous avons nommé fréquence relative, pour une période donnée et pour un stade donné (adulte, juvénile), le rapport entre le nombre d'échantillons avec des éperlans et le nombre total d'échantillons. Ce paramètre de type présence-absence nous a semblé intéressant dans le cas de l'analyse du fonctionnement de la population d'éperlan car:

- il est très rustique (insensible aux estimations des volumes filtrés et aux dénombrements) ;

- il est adapté aux espèces présentes seulement durant certaines périodes de l'année ;

- il permet de mettre en évidence des évolutions tranchées.

\section{Calcul des abondances}

Les abondances ont été estimées pour chaque prélèvement de façon simple grâce à une relation $\left(E_{1}\right)$ qui relie les volumes filtrés par les engins de pêche et le nombre d'individus capturés :

$$
\hat{V}=\frac{(n \times 1000)}{V}
$$

$\hat{N}$ : estimation de l'abondance en nombre d'individus $/ 1000 \mathrm{~m}^{3}$ d'eau filtrée,

$n$ : nombre d'individus capturés,

$V$ : volume filtré pendant le prélèvement. 
En fonction des besoins, des indices d'abondance pourront être construits à un pas de temps mensuel ou annuel, ou pour une période particulière, en calculant la moyenne des estimations d'abondance pour la période considérée.

\section{Relation stock-recrutement}

II s'agit d'examiner la relation entre l'abondance des géniteurs et celle de leur progéniture au cours de la période d'étude. Etant donné que nous sommes en présence d'une espèce migratrice, qui n'est pas présente en permanence sur le site, pour estimer ces abondances nous avons préalablement défini les périodes correspondant au pic d'abondance de chacune des deux écophases. Pour cela, nous avons exprimé les estimations d'abondance de chaque mois de façon relative, en pourcentage de l'abondance maximale estimée.

Nous avons alors mis en relation l'abondance en géniteurs d'éperlans $A_{g}$ et en recrues d'éperlans $A_{n}$, à savoir la somme des densités mensuelles moyennes des éperlans de l'écophase considérée, capturés durant la période correspondant au pic d'abondance.

La population de l'estuaire de la Gironde est petite comparativement à la taille du bassin versant, y compris au début de la période analysée. Nous considérons donc que nous nous situons dans la partie initiale de la relation stock-recrutement reliant les effectifs des adultes à leur progéniture et que nous pouvons l'assimiler à une droite $\left(E_{2}\right)$ passant par l'origine dont nous avons calculé la pente $\alpha$ :

$$
A_{r}=a \times A_{g}
$$

Nous pouvons ainsi, si nous disposons d'une estimation non biaisée de l'abondance des géniteurs, estimer ce que pourrait être l'abondance de leur progéniture à l'aide de cette relation. Le rapport entre les effectifs observés et les effectifs prédits par la régression linéaire constitue un résidu, noté $\varepsilon$. Dans cette relation nous considérons que le résidu est un facteur car nous avons retenu un modèle de type multiplicatif.

$$
\begin{aligned}
A_{r}(o b s) & =\left(a \times A_{g}\right) \times \varepsilon \\
\text { d'où } & \varepsilon=\frac{A_{r}(o b s)}{A_{r}(c a l c)}
\end{aligned}
$$

En première approche, nous avons choisi d'établir une relation incluant une régulation densité dépendante, considérant que nous sommes dans le cas d'une espèce se reproduisant en eau douce et utilisant des habitats dont la capacité d'accueil est limitée. Nous avons, comme cela a été fait pour d'autres espèces amphihalines (MARTIN, 1996), choisi une relation de type RICKER $\left(E_{4}\right)$.

$$
A_{r}=\alpha A_{g} e^{-\left(\frac{A_{g}}{K}\right)}
$$

\section{Evaluation du taux de mortalité}

II existe une mortalité accessoire induite par la pêcherie de crevette blanche Palaemon longirostris (CASTELNAUD, 1978 ; CASTELNAUD et ROCHARD, 1996) dans l'estuaire de la Gironde. C'est pourquoi nous considérons que ce que nous estimons correspond à la mortalité totale $Z$ dont nous avons calculé le taux instantané selon deux approches.

Pour chaque cohorte, nous avons déterminé le pic d'abondance au stade juvénile en estuaire et nous avons estimé un taux de mortalité instantané $Z_{1}$ sur la période située entre 
ce pic $\left(t_{p l c}\right)$ et 3 mois plus tard $\left(t_{p 1 c}-3\right)$. Nous considérons que cette décroissance est exclusivement causée par la mortalité à l'exclusion de toute migration en dehors de l'estuaire.

Les valeurs d'abondance $\left(N_{\text {tpicc }}, N_{\text {tpict }+1}, \ldots N_{\text {pic }+3}\right)$ ont été transformées en leur logarithme de façon à rendre le modèle additif et à visualiser la décroissance, le taux de mortalité $Z_{1}$ a alors été estimé par la pente de cette droite $\left(E_{5}\right)$.

$$
\operatorname{Ln} N_{t^{\prime}}=\left(-Z_{1}\right)^{\star} t^{\prime}+b
$$

Selon le même principe que précédemment, nous avons estimé pour chaque cohorte un taux de mortalité instantané $Z_{2}$ entre l'abondance au stade juvénile (moyenne des $N_{j}$ sur la période située entre le pic $\left(t_{\text {pic }}\right)$ et 3 mois plus tard $\left(t_{\text {pic }}+3\right)$ ) et l'abondance de la même cohorte au stade adulte (moyenne des $\mathrm{N}_{\mathrm{a}}$ sur la période de densité maximale).

Ces deux taux de mortalité peuvent être mis en relation avec la température de façon à évaluer son influence sur le stade juvénile seul ou plus globalement sur l'ensemble du cycle de vie de l'animal. On gardera cependant à l'esprit qu'il s'agit d'estimations d'abondance dont la précision n'est pas connue et que par ailleurs, il s'agit d'une espèce dont nous connaissons encore mal les patrons de migration.

\section{RÉSULTATS}

Sur la période considérée (18 années), nous avons effectué 4063 prélèvements, 2053 en surface ( 2751 adultes et 15910 juvéniles capturés) et 2010 en fond ( 2611 adultes et 14408 juvéniles). L'ensemble de la période a été échantillonnée conformément au protocole (sans lacunes de plus d'un mois consécutif) à l'exception notable de deux périodes (juin-juilletaoût 1981) et (février-mars-avril 1989) pour lesquelles aucun prélèvement n'a été effectué. Pour cette raison, ces deux années seront exclues de certains traitements et analyses.

\section{Caractéristiques thermiques}

Sur la période d'étude, la température de l'eau montre une tendance interannuelle nettement à la hausse (Figure 3). Ceci est confirmé par les suivis météorologiques atmosphériques réalisés par Météo France. Le maximum a été atteint en 1989-1990 avec un gain d'environ $2,0^{\circ} \mathrm{C}$ par rapport à 1979 . Le Tableau II indique les années correspondant à chacun des 4 grands types thermiques que nous avons définis. On constate une assez forte variabilité interannuelle avec simplement deux périodes homogènes (1982-1984) et (1985-1987).

Les prélèvements ont été réalisés dans une gamme thermique allant de $1,0^{\circ} \mathrm{C}$ (Hiver 1985) à $29,5^{\circ} \mathrm{C}$ (Eté 1982). Les éperlans n'ont été capturés que dans un intervalle allant de $4,5^{\circ} \mathrm{C}$ à $29,5^{\circ} \mathrm{C}$ pour les juvéniles et de $5,0{ }^{\circ} \mathrm{C}$ à $29,5^{\circ} \mathrm{C}$ pour les adultes, soit des températures extrêmement élevées pour l'espèce. Lors de 7 épisodes, des éperlans ont été capturés au-delà de $25^{\circ} \mathrm{C}$ (86 prélèvements) et lors de 3 épisodes des éperlans ont été capturés en dessous de $5^{\circ} \mathrm{C}(34$ prélèvements).

\section{Fréquence de l'espèce}

La fréquence de l'éperlan, tous stades confondus, sur l'ensemble de la période est de $48 \%$ (24\% pour les adultes et $38 \%$ pour les juvéniles). Pour les adultes, l'évolution interannuelle (Figure 4) montre une décroissance régulière depuis le début du suivi jusqu'en 1991, puis une augmentation de 1994 à 1996 . Pour les juvéniles, entre 1979 et 1987, la fréquence fluctue (Figure 4) mais demeure au-delà de $50 \%$, elle chute de 1988 à 1990 avant d'augmenter comme pour les adultes à partir de 1994. De 1979 à 1982, l'écart entre la fréquence relative des deux stades est minime $(<10 \%)$, il augmente ensuite considérablement avec un maximum en 1985. 


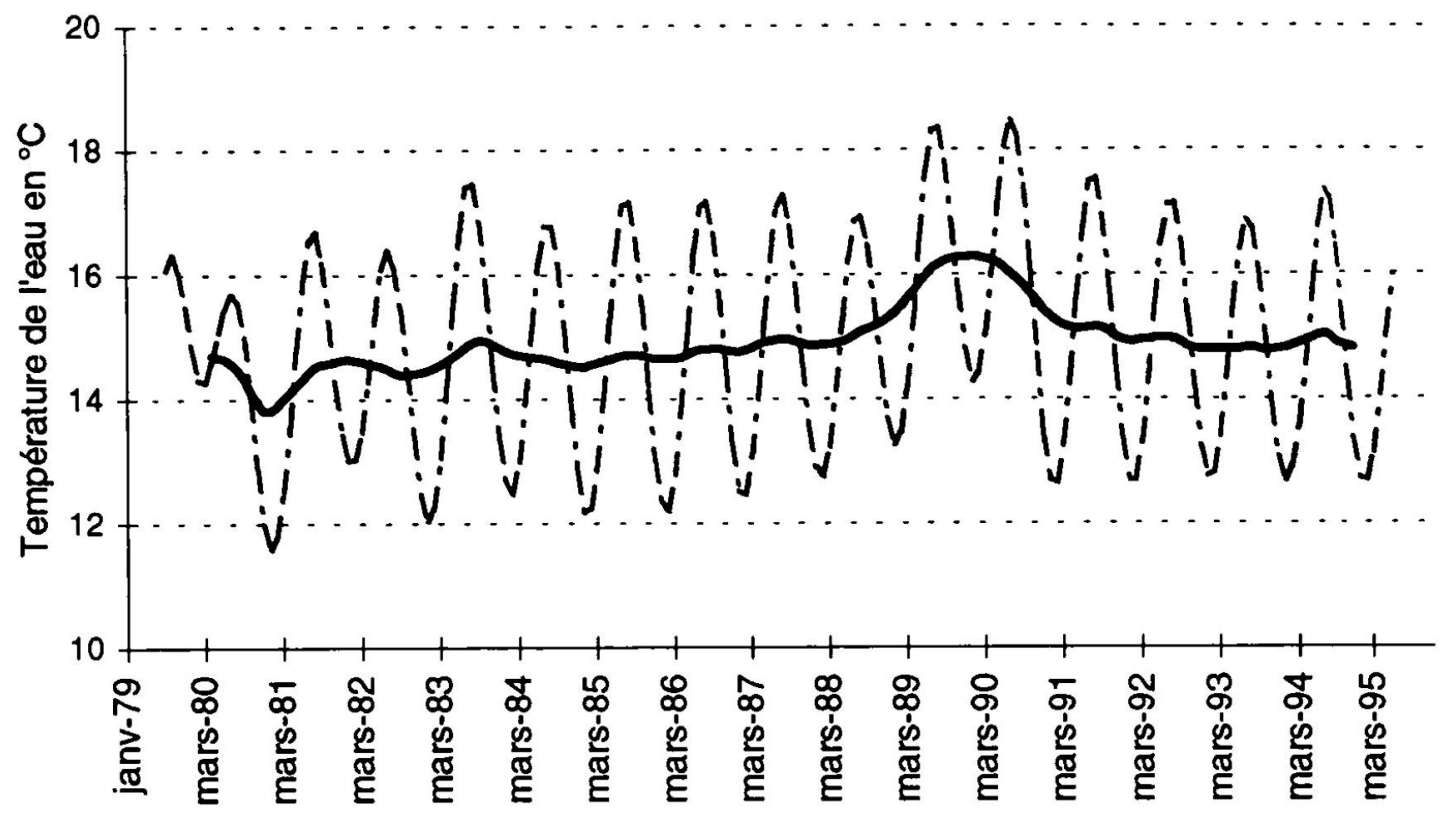

Figure 3

Evolution temporelle de la température de l'eau de l'estuaire de la Gironde en ${ }^{\circ} \mathrm{C}$. Série chronologique pondérée par une moyenne mobile sur 13 mois (tiretés) et 25 mois (traits pleins).

Figure 3

Evolution of the Gironde estuary water temperature in ${ }^{\circ} \mathrm{C}$. Time series weighted with a moving average on 13 months (dot lines) and 25 months (bold lines).

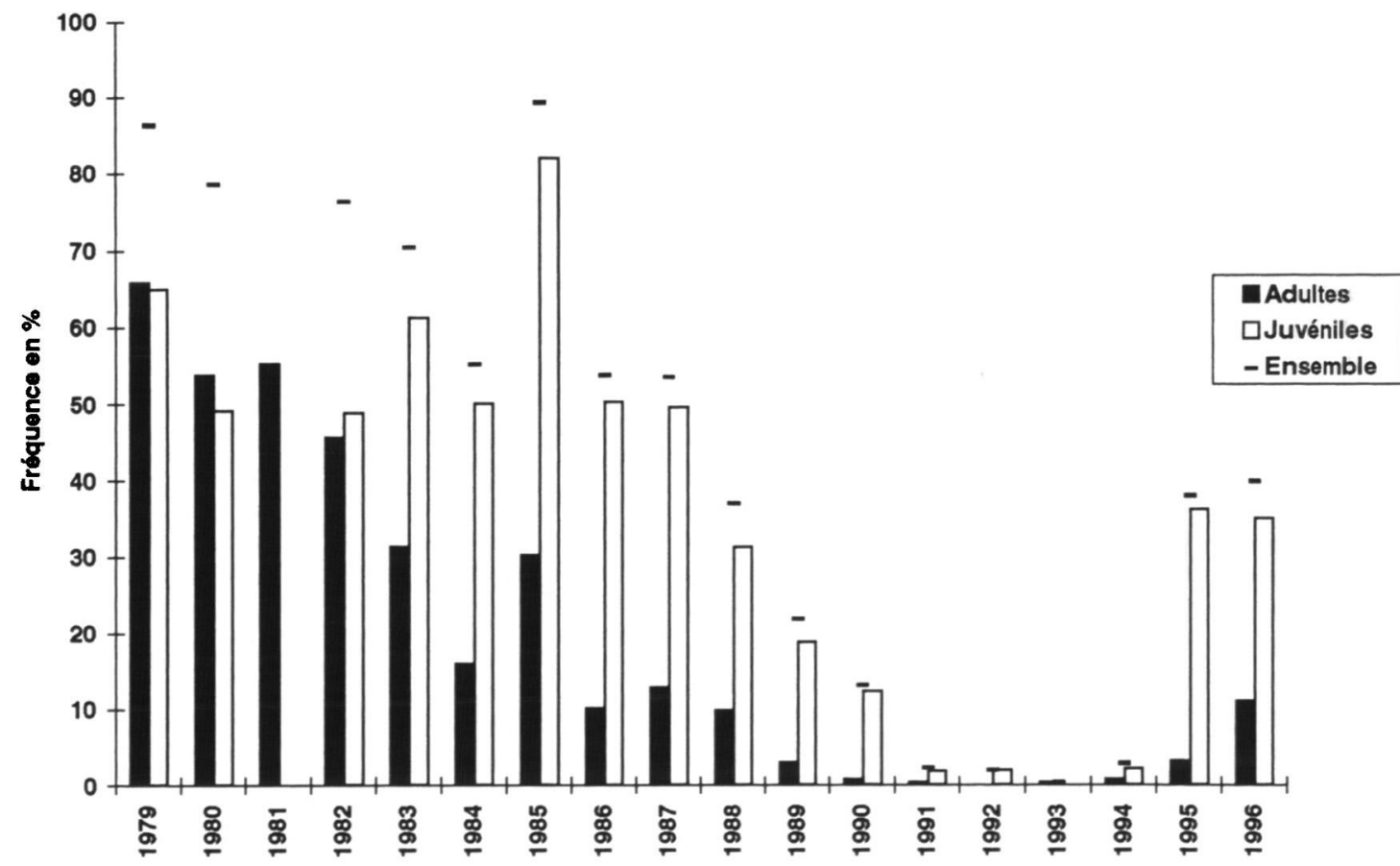

Figure 4

Fréquence relative des prélèvements avec présence d'éperlan (en \%).

Figure 4

Related frequency of samples with smelt (in \%). 


\section{Evolution de l'abondance}

Globalement on observe une nette diminution des abondances entre 1979 (densité moyenne annuelle de $10,86 \mathrm{ind} / 1000 \mathrm{~m}^{3}$ ) qui représente le maximum de la série et la période 1989-1993 (densité moyenne annuelle extrêmement faible voire nulle) qui en constitue le minimum (Figure 5). A partir de 1994, on remarque une recrudescence des abondances. La proportion d'adultes est en moyenne sur l'ensemble de la période de $16,35 \%$, mais présente de fortes variations d'une année à l'autre.

Concernant les abondances au stade adulte on observe, comme pour les fréquences, une décroissance régulière sur l'ensemble de la période 1979-1993. L'évolution de l'abondance des juvéniles est beaucoup moins nette, on note deux pics équivalents, en $1979\left(9,36 \mathrm{ind} / 1000 \mathrm{~m}^{3}\right)$ et $1982\left(9,26 \mathrm{ind} / 1000 \mathrm{~m}^{3}\right)$ puis une période $1989-1994$ avec des abondances nulles ou quasiment.

Cette quasi-disparition des éperlans en Gironde s'avère concomitante avec l'augmentation de la température de l'eau (Figure 3).
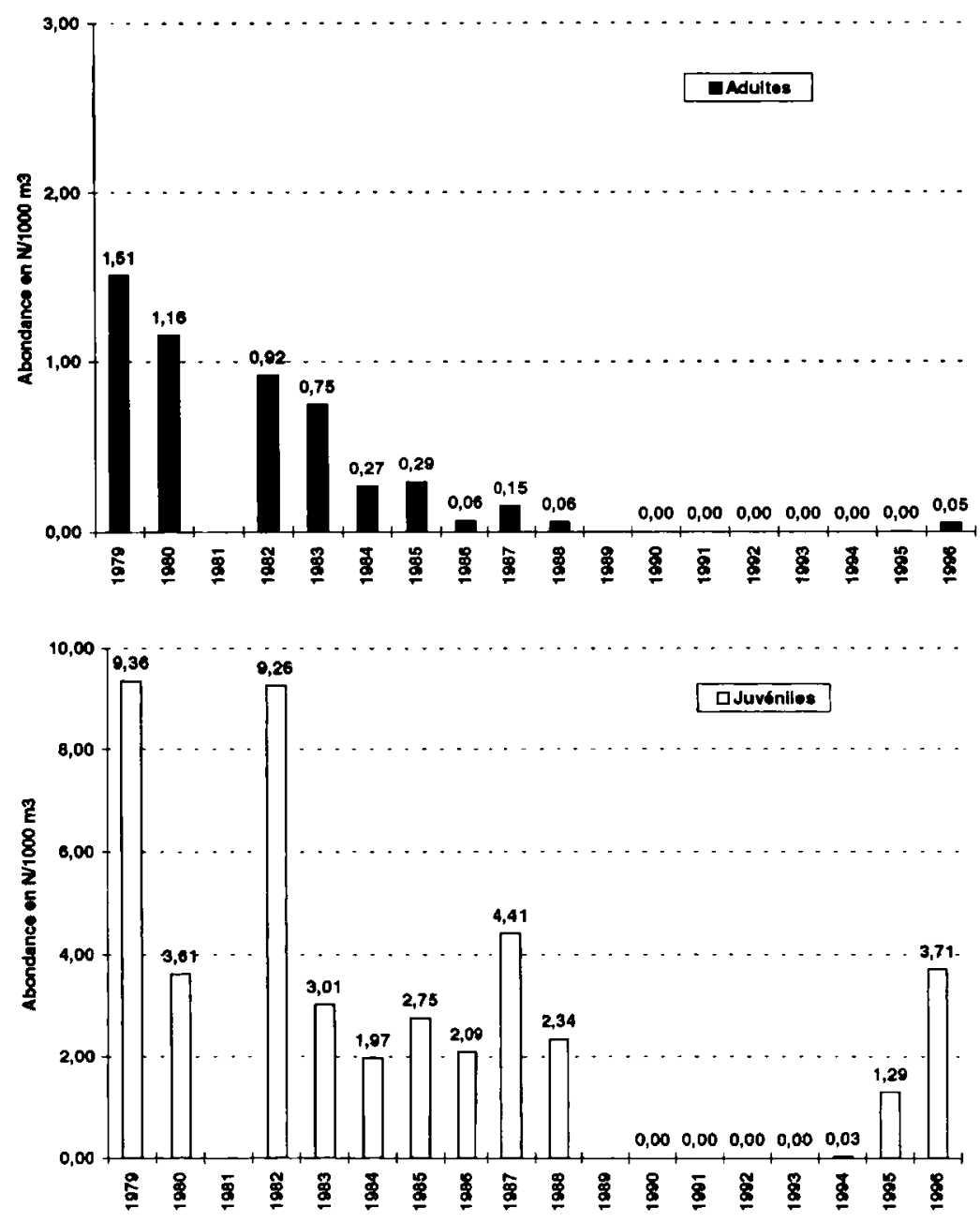

\section{Figure 5}

Evolution de l'estimation de l'abondance d'éperlans entre 1979 et 1995 (en N/1000 m³).

\section{Figure 5}

Evolution of smelt abundance estimation in Gironde between 1979 and 1995 (Number/1000m ${ }^{3}$ ). 


\section{Périodes d'abondance}

Les abondances relatives les plus élevées en éperlans adultes (Figure 6) se situent au début de l'année (janvier-avril) au moment où les géniteurs traversent l'estuaire pour aller ou revenir des zones de frayères situées dans la partie basse des fleuves. Les juvéniles, quant à eux, arrivent en estuaire à partir de mai et montrent un pic d'abondance en moyenne situé en juin-juillet. La période retenue pour l'estimation de l'abondance des géniteurs est janvier-avril, pour les juvéniles en raison d'une plus forte variabilité interannuelle nous considérerons une période de 3 mois à partir du pic de chaque année.

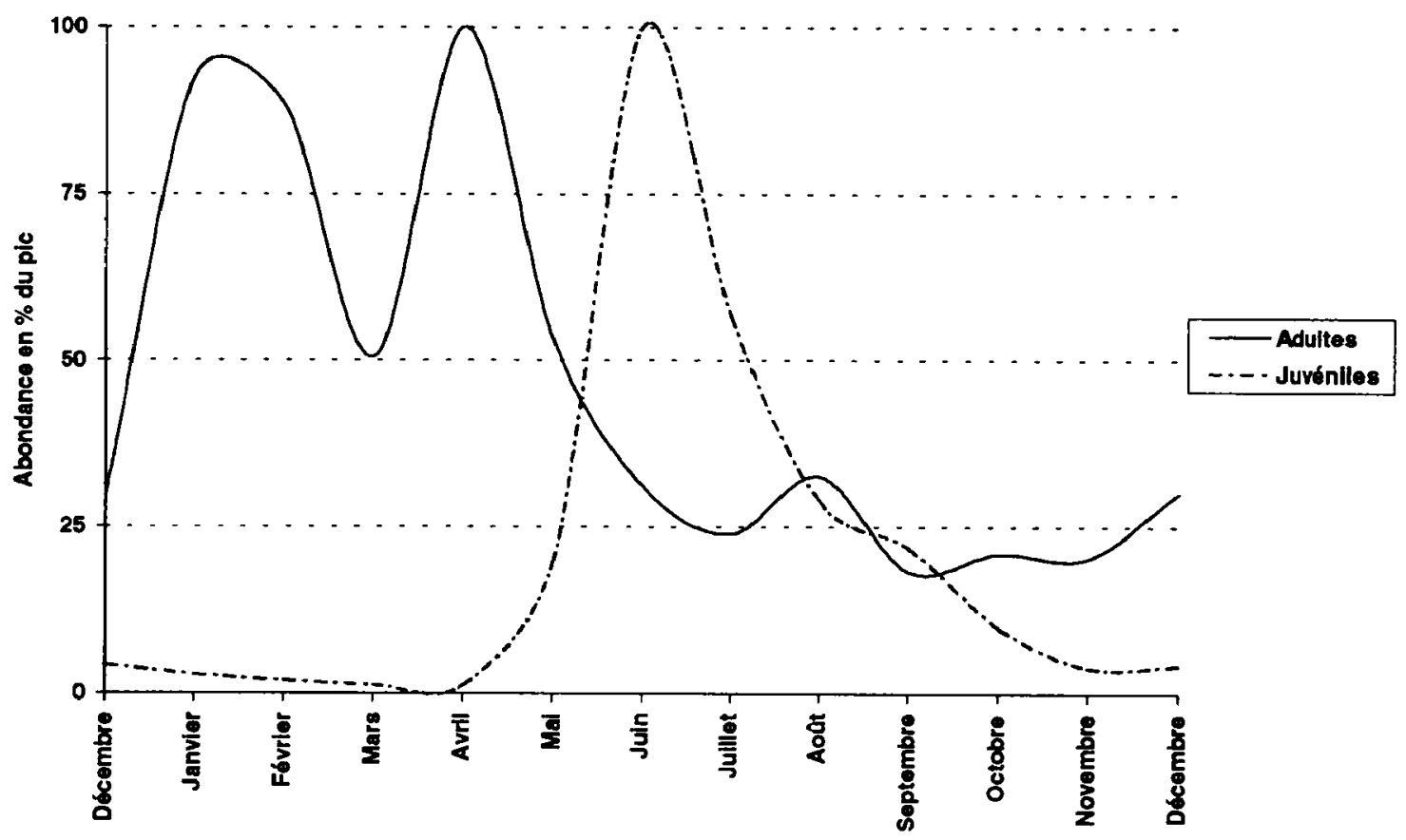

Figure 6

Abondance relative moyenne sur l'ensemble de la période d’étude (en \% du pic).

\section{Figure 6}

Related mean abundance all over the studied period (in \% of the maximum).

\section{Relation stock-recrutement}

La Figure 7 met en évidence deux groupes de points relativement disjoints, l'un avec de fortes valeurs d'abondance correspondant au début de la période de suivi et l'autre avec de faibles valeurs d'abondance correspondant à la fin de la période. Si on les considère globalement, on peut en établir l'équation linéaire $\left(E_{2}\right) A_{r}=6,476 * A_{g}+9,013$ (avec $R^{2}=0,79$ ) correspondant cependant uniquement à la partie initiale de la relation stock-recrutement.

Le point correspondant à l'année $1983\left(A_{g}=8,96, A_{r}=17,16\right)$ pose par contre un problème d'interprétation, il correspond en effet à une abondance en géniteurs élevée mais à une faible abondance de recrues. Si la valeur de l'estimation est bonne (non biaisée et même précision que les autres années) cela pourrait correspondre à la partie droite de la courbe $S / R$, en revanche si elle est aberrante cela signifierait que l'on se trouve encore dans la partie initiale de la relation stock-recrutement, que le phénomène est très variable et qu'on ne peut pas établir de relation générale. 


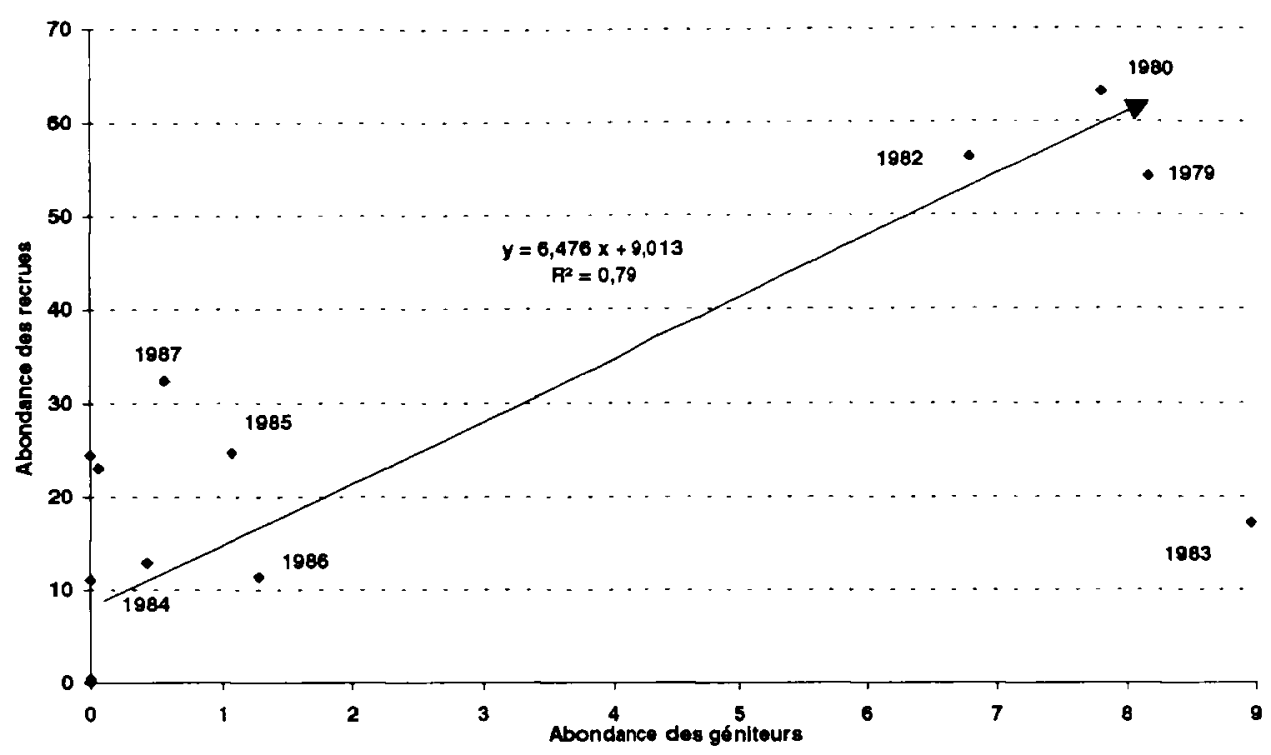

Figure 7

Relation stock-recrutement entre l'abondance des géniteurs ( $\mathrm{Ag}$ ) et celle de leur progéniture (Ar), équation et coefficient de détermination de la droite de régression (année 1983 exclue). L'abondance des géniteurs est estimée par la somme (janvier-avril) des moyennes mensuelles des abondances et celle de leur progéniture par la somme des moyennes mensuelles sur 3 mois à partir du pic d'abondance de la cohorte.

\section{Figure 7}

Stock-recruitment relationship between broodstock abundance (Ag) and fry one (Ar), equation and determination coefficient of the regression curve (except for 1983). The broodstock abundance is assessed using the sum of the monthly mean abundance and the fry abundance is assessed using the sum of the 3 monthly mean abundances from the cohort abundance maximum.

En ce sens, on peut considérer qu'il correspond à des conditions environnementales particulières ou bien qu'il indique un phénomène compensateur très fort de type densité dépendant. En tout état de cause, l'année 1983 correspond pour la relation stockrecrutement à une situation de rupture entre deux périodes correspondant à des situations différentes de la population. Pourtant, au point de vue thermique l'année 1983 est du même type que ses voisines 1982 et 1984 (hiver standard et été chaud).

L'équation de RICKER $\left(E_{4}\right)$ correspondant à notre jeu de données est la suivante :

$$
A_{r}=5,937 \times A_{g} e^{\cdot(A g ; 8,995)}
$$

En admettant que dans la gamme des valeurs observées on puisse rencontrer une relation densité dépendante, l'abondance en géniteurs correspondant au maximum de la courbe se situerait à $A_{g}=19,54$ ind $/ 1000 \mathrm{~m}^{3}$ soit bien au-delà de ce que nous avons observé pour l'année 1983.

La distribution des résidus $\varepsilon$ issus de $\left(E_{3}\right)$ (Tableau II) ne montre pas de lien par rapport à la température estivale, ni par rapport à la température moyenne de l'année précédente. On remarque simplement que les résidus situés en dessous de 1 (valeur observée plus faible que la valeur issue de $\left(E_{2}\right)$ ) correspondent aux températures moyennes hivernales (moment de la reproduction) les plus froides. Par contre, si l'on examine ces 
mêmes résidus par rapport au type thermique de ces années, cela ne correspond pas à un type particulier. II ne semble pas y avoir de relation significative entre la température et les résidus de la relation stock-recrutement.

\section{Tableau II}

Caractéristiques des différentes années. Types thermiques $(1$ : hiver froid - été standard, 2 : hiver standard - été standard, 3 : hiver standard - été chaud, 4 : hiver froid - été chaud). Température moyenne hivernale (janvier-avril), estivale (juinseptembre) et annuelle en ${ }^{\circ} \mathrm{C}$. Taux de mortalité instantanés pour les juvéniles (Z1) et entre les juvéniles et les adultes d'une même cohorte (Z2). $\varepsilon$ : résidus correspondant à la relation stock-recrutement, issus de la relation (E4).

Table II

Characteristics of the different years. Thermal types $(1$ : cold winter - standard summer, 2 : standard winter - standard summer, 3 : standard winter - warm summer, 4 : cold winter - warm summer). Average temperature for winter (JanuaryApril), summer (June-September) and all the year in ${ }^{\circ} \mathrm{C}$. Instantaneous rates of mortality for the juveniles (Z1) and between juveniles and spawners of the same cohort (Z2). $\varepsilon$ : residuals from the stock-recruitment relationship, from (E4).

\begin{tabular}{|c|c|c|c|c|c|c|c|c|c|c|c|c|c|c|c|c|c|c|}
\hline Annoes & 1979 & 1980 & 1981 & 1982 & 1983 & 1984 & 1985 & 1986 & 1987 & 1988 & 1989 & 1990 & 1991 & 1992 & 1993 & 1994 & 1995 & 1996 \\
\hline Type thermique & & 2 & & 3 & 3 & 3 & 1 & 1 & 1 & 2 & & & 4 & 2 & 2 & 1 & 2 & 2 \\
\hline$T^{\circ}$ hivernale & & 9,19 & & 10,44 & 8,87 & & 8.93 & 9.04 & 8,97 & 10,21 & 9,38 & 10,11 & 8,84 & 8,86 & 9,96 & 7,91 & & \\
\hline To estivale & 20,5 & 18,9 & & 22,6 & 21,9 & 20,5 & 22,2 & 21,1 & 21,8 & 20,4 & & & 23,3 & 21,4 & 19,9 & 20,1 & 17,6 & 19,4 \\
\hline$T^{\circ}$ annuelle & 14,4 & 13,4 & & 15,6 & 14,9 & 14,9 & 15,0 & 14,8 & 15,0 & 14,7 & & & 15,4 & 14,8 & 13,7 & 14,2 & 14,6 & 13.2 \\
\hline$Z_{1}$ & 0,17 & 0,26 & & 0,48 & 0,24 & 0,26 & 0,57 & 0,37 & 0,35 & 0,55 & & & & & & & 0,59 & 0,47 \\
\hline$z 2$ & 0,84 & 0,47 & & 0,80 & 4,13 & 1,02 & 1,76 & 1,37 & & & & & & & & & 2,26 & 1,21 \\
\hline$\varepsilon$ & 0,87 & 1,06 & & 1,06 & 0,26 & 0,66 & 1,55 & 1,09 & 2,57 & 2,71 & & & & & & 0,04 & 1,22 & 2,45 \\
\hline
\end{tabular}

\section{Mortalité}

Le taux de mortalité instantané $Z_{1}$ sur la période de présence initiale des juvéniles en estuaire est en moyenne de 0,396 (min. 0,174, max. 0,597). L'évolution interannuelle de ce

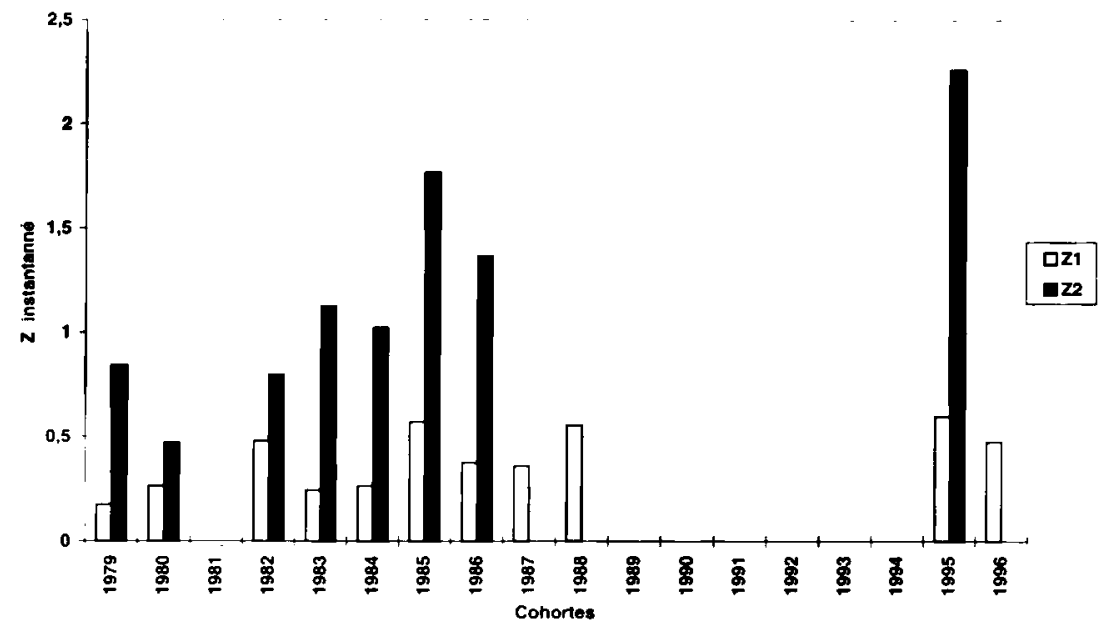

Figure 8

Taux de mortalité instantanés pour les juvéniles $(Z 1)$ et entre les juvéniles et les adultes d'une même cohorte (Z2).

\section{Figure 8}

Instantaneous rates of mortality for the juveniles $(Z 1)$ and between juveniles and spawners of the same cohort (Z2). 
paramètre (Figure 7) met en évidence une très légère augmentation entre 1979 et 1988 , mais fait surtout ressortir des années avec des mortalités un peu plus élevées $(1982,1985$ $1988,1995,1996)$, sans pour autant qu'elles soient du même type thermique.

Les valeurs des mortalités $Z_{2}$ sur l'ensemble des années pour lesquelles les calculs ont pu être effectués sont en moyenne de 1,206 ( $\min .0,471$, max. 2,256) et montrent une tendance nette à l'augmentation au cours du temps, soit un parallèle très étroit avec l'augmentation moyenne de la température. On ne trouve par contre aucun lien entre $Z_{2}$ et les températures moyennes estivales ou hivernales.

\section{DISCUSSION}

\section{Température}

Nous avons mis en évidence une augmentation de la température de l'eau de l'estuaire de la Gironde au cours de la période d'étude, les données atmosphériques confirment un réchauffement global du sud-ouest de la France. Dans notre cas, l'installation au début des années $\mathbf{8 0}$ de la centrale nucléaire du Braud et St-Louis, refroidie par l'eau de l'estuaire, n'a pu que participer à ce phénomène, sans que nous puissions identifier sa contribution. Dans une zone de moins de 3 ha autour du rejet de l'eau ayant servi au refroidissement de la centrale, la température de l'eau est de $3^{\circ} \mathrm{C}$ plus élevée que la température normale de l'estuaire. La courbe d'isoéchauffement $1^{\circ} \mathrm{C}$ enveloppe au maximum une surface de $55 \mathrm{~km}^{2}$ (BOIGONTIER et MOUNIÉ, 1984). II serait intéressant d'obtenir des données thermiques en amont de la centrale sur la même période afin d'estimer l'influence de la centrale sur la température de l'eau à son niveau. Malheureusement, il n'existe pas à notre connaissance de chroniques précises de température disponibles.

\section{Situation de la population}

L'évolution de la fréquence de l'éperlan (Figure 4) montre une diminution très nette, elle est particulièrement régulière pour le stade adulte. Les éperlans sont donc rencontrés plus rarement dans l'estuaire de la Gironde que précédemment, que ce soit parce que leurs effectifs sont en baisse ou parce qu'ils ne reviennent pas dans cet estuaire après leur passage en mer. Cette « disparition » progressive est d'abord observée chez les adultes puis chez les juvéniles, elle est telle qu'à un moment l'espèce passe en dessous du seuil de détection. Les années les plus récentes (1995 et 1996) montrent une « réapparition » de l'espèce mais à un niveau encore faible.

Dans la mesure où nous ne disposons pas d'une bonne appréciation de la précision de notre estimateur d'abondance, les valeurs doivent être interprétées avec prudence et uniquement pour des évolutions nettes.

L'évolution de l'abondance du stade adulte de l'éperlan (Figure 5) montre une diminution régulière sur toute la période d'étude, il est possible qu'elle ait commencé auparavant mais nous ne disposons pas d'élément sur la période précédente.

L'évolution des abondances du stade juvénile montre entre 1979 et 1988 des fluctuations fortes, ce qui est relativement classique pour cette famille de petits poissons pélagiques à cycle court pour lesquelles les fluctuations sont souvent liées aux fluctuations environnementales (MOYLE et al., 1992). Par ailleurs, sur la période 1989-1994 l'abondance est nulle ou quasiment. On remarque que tant que l'abondance des géniteurs est demeurée au-dessus d'un certain seuil (qui pourrait se situer autour de 0,05 ind/1000 $\mathrm{m}^{3}$ ) l'abondance des juvéniles tout en étant variable est restée au-dessus de 2 ind $/ 1000 \mathrm{~m}^{3}$, par contre dès 
que l'abondance des géniteurs est descendue en dessous de ce seuil cela s'est traduit immédiatement pour les juvéniles par un passage en dessous du seuil de détection.

Les valeurs très faibles enregistrées de 1989 à 1994 correspondent au seuil de détectabilité de l'espèce par les moyens mis en oeuvre. En effet, compte tenu de la taille de la zone étudiée, il serait abusif d'affirmer que l'espèce ait complètement disparu durant cette période. Si cela était le cas, la remontée des abondances que l'on observe en 1995 ne serait alors explicable qu'à partir d'une recolonisation allochtone, par exemple à partir de la Loire.

L'analyse historique de la présence de l'éperlan dans l'estuaire de la Gironde montre que cette espèce a déjà montré des phases d'abondance succédant à des quasi-disparitions. Ainsi, dans les croyances des pêcheurs actuels l'éperlan est une espèce qui est apparue après la Seconde Guerre mondiale, alors qu'elle était pourtant déjà présente au milieu du $19^{\mathrm{e}}$ siècle dans la faune du département de la Gironde (LAPORTE, 1853).

Au bilan, entre le début et la fin de la période d'étude, et de façon concomitante avec la hausse de la température, les éperlans ont été rencontrés de moins en moins fréquemment dans l'estuaire de la Gironde. De plus lorsqu'ils ont été rencontrés, ils étaient alors en plus faible abondance. Dans les deux cas, l'évolution visualisée sur le stade adulte est plus nette que pour le stade juvénile.

\section{Mécanisme de la diminution}

La relation stock-recrutement que nous avons établie ne permet pas d'identifier d'anomalie dans le fonctionnement de la population, ni même de tendance en liaison avec la température, à l'exception notoire de l'année 1983 pour laquelle le recrutement a été nettement plus faible qu'attendu. Au niveau thermique, l'année 1983 ne se distingue pas nettement des années voisines, la cause de ce faible recrutement est certainement ailleurs. Cette forte variabilité est communément observée chez les petits pélagiques (MENDELSSOHN et CURY, 1987). Contrairement à ce qui a été mis en évidence sur certains bassins versants fréquentés par Osmerus mordax (ROBITAILLE et VIGNEAULT 1990 ; LYLE et MAITLAND, 1997), aucune modification drastique des caractéristiques physiques des zones de reproduction et de développement des oeuts n'a été remarquée au cours de la période d'étude.

Ceci nous amène à penser que l'évolution de la population ne serait pas liée à une action de l'environnement sur la reproduction mais plutôt de façon diffuse sur la survie entre le stade juvénile et le stade adulte.

L'estimation du taux de mortalité instantané sur la période de présence initiale des juvéniles $\left(Z_{1}\right)$ ne montre pas de tendance évolutive nette, par contre l'estimation du taux de mortalité entre le stade juvénile et le stade adulte $\left(Z_{2}\right)$ montre une augmentation régulière sur l'ensemble de la période et en parallèle avec l'augmentation de la température.

On peut donc supposer que la diminution de la population soit plutôt due à une action diffuse entraînant une mortalité (ou un non-retour des géniteurs dans le bassin versant) progressivement de plus en plus importante, qu'à une action ponctuelle (que ce soit par des températures estivales élevées ou des températures hivernales extrêmes).

On peut cependant évoquer une hypothèse complémentaire. En effet, il est connu qu'en limite de zone de tolérance pour la température la croissance se ralentit (JOBLING, 1997), ceci en liaison avec un ralentissement de l'alimentation (MAC CARTHY et HOULIHAN, 1997).

Il s'avère que la taille maximale des éperlans rencontrée en Gironde est inférieure à $200 \mathrm{~mm}$ (Lt.) soit nettement plus faible que celle des populations situées au coeur de l'aire 
de répartition, ceci est cohérent à la fois avec une mortalité importante qui entraîne la mort des animaux avant qu'ils aient atteint cette taille et avec une croissance plus faible.

Pour une espèce donnée, l'âge à la première reproduction est le plus fréquemment, taille-dépendant. Si la croissance des éperlans de Gironde ralentit, cela entraîne par conséquent une diminution de la fraction des cohortes qui va pouvoir se reproduire à partir de 1 an aux dépens de celle qui va se reproduire à partir de 2 ans. Ceci s'accompagne par conséquent d'une diminution des effectifs de géniteurs.

On aboutirait ainsi à une modification thermo-dépendante du cycle écologique avec un âge à la première reproduction qui passerait de 1 an à 2 ans. Un mécanisme de ce type a été évoqué récemment pour le saumon atlantique Salmo salar (MAC CARTHY et HOULIHAN, 1997). Pour l'éperlan le scénario peut se résumer de la façon suivante:

- une action négative de la température sur la croissance des individus entraînant

:: recul d'une saison de l'âge à la première maturité sexuelle

: diminution du nombre de géniteurs

- une action négative de la température sur la survie entraînant

$\therefore$ diminution des effectifs de la population

Mais on ne peut pas non plus exclure que le phénomène que nous avons mis en évidence s'insère en fait dans un cycle de variation des effectifs d'amplitude beaucoup plus importante, et dont les causes sont antérieures à l'étude voire strictement liées à une dynamique complexe encore mal appréhendée.

II serait intéressant de compléter cette approche par l'examen de la covariation d'autres facteurs environnementaux susceptibles d'expliquer certaines particularités de cette dynamique, par exemple la valeur très faible du recrutement de 1983.

Pour la suite du suivi de l'ichthyofaune de l'estuaire de la Gironde, nous préconisons de prendre en compte des facteurs biologiques importants tel l'âge ou le sexe, qui permettrait une meilleure interprétation de la dynamique et de mener un travail de fond sur la précision des estimateurs utilisés.

\section{CONCLUSION}

La population d'éperlan de l'estuaire de la Gironde se situe en limite méridionale de son aire de répartition. Cette particularité correspond à une relative fragilité vis-à-vis du réchauffement de son environnement.

Durant la période d'étude (1979-1996) nous avons constaté une élévation progressive de la température de l'eau de l'estuaire avec un maximum en 1989-1990. Pendant le même temps la fréquence de rencontre de l'éperlan et l'abondance de la population (telle que nous l'avons appréhendée) ont diminué jusqu'à passer pendant plusieurs années en dessous du seuil de détectabilité. Cette diminution est beaucoup plus nette et régulière pour le stade adulte que pour le stade juvénile.

Dans notre jeu de données, la relation stock-recrutement apparaît très difficile à interpréter. Notre analyse basée sur une relation stock-recrutement linéaire ne permet pas de mettre en évidence une action négative de la température sur la reproduction, une approche visant à explorer une relation non linéaire entre les géniteurs et les juvéniles pourrait être intéressante bien que nécessitant un jeu de données plus consistant (CURY, 1995). Par contre, la mortalité entre le stade juvénile et le stade adulte augmente en même temps que la température. 
II nous semble que l'hypothèse la plus probable soit celle d'une action complexe de la température sur la dynamique de cette population par l'intermédiaire du ralentissement de la croissance, ceci entraînant un basculement d'un cycle écologique avec une reproduction à 1 an à un cycle avec une reproduction à 2 ans.

\section{REMERCIEMENTS}

Ce travail a été réalisé dans le cadre d'un contrat de collaboration entre le Cemagref et la Direction Etudes et Recherches de EDF. Notre pensée va à Jacques CASTEL (†, Institut Universitaire de Biologie Marine d'Arcachon) qui nous a encouragé dans la réalisation de cette étude. Nous remercions François TRAVADE pour l'intérêt qu'il a porté au sujet et pour son soutien stimulant durant toute la durée de sa réalisation, Patrick LAMBERT pour ses remarques et ses commentaires toujours pertinents et Gilles ADAM pour l'aide apportée dans l'utilisation des bases de données. Nous sommes reconnaissants à Patrick PROUZET (Ifremer Saint-Pée-sur-Nivelle) et à un référé anonyme pour leurs critiques constructives et leur aide à la révision de ce document. Nous tenons également à associer à ce travail tous ceux et celles qui depuis le début ont contribué d'une façon ou d'une autre à la réalisation du suivi halieutique de l'estuaire de la Gironde.

\section{BIBLIOGRAPHIE}

ANONYME, 1979. Etude de surveillance halieutique de l'estuaire de la Gironde. Rapp. Tech. Cemagref/EDF, $90 \mathrm{p}$.

ALTUKHOV K.A., YERASTOVA V.M., 1974. Reproduction of smelt (Osmerus eperlanus) and the ecology of its early development stages in Kandalaksha bay. White Sea J. of Ichtyol., 14 (6), 877-886.

BOIGONTIER B., MOUNIÉ D., 1984. Contribution à la connaissance de la dynamique de la macrofaune bentho-démersale et pélagique en Gironde. Tentatives et difficultés pour relativiser l'impact mécanique d'une centrale nucléaire : Le Blayais (Gironde). Thèse de Doctorat de 3ème cycle, ENSA Toulouse/Cemagref de Bordeaux, Div. A.L.A., 491 p.

BORCHARDT D., 1988. Long-term correlations between the abundance of smelt (Osmerus eperlanus $L$.) year classes and abiotic environmental conditions during the period of spawning and larval development in the Elbe river. Arch. Fisch. Wiss., 38 (3), 191-202.

CASTELNAUD G., 1978. Etude de la pêche aux filets et aux engins dans l'estuaire de la Gironde. Thèse de Doctorat de 3ème cycle. Cemagref de Bordeaux, Div. A.L.A./Université de Bordeaux III, 198 p.

CASTELNAUD G., ROCHARD E., 1996. Surveillance halieutique de l'estuaire de la Gironde. Suivi statistique 1994, étude de la faune circulante 1995. Cemagref de Bordeaux, étude $n^{\circ}$ 15/EDF, $158 p$.

COX D.K., 1974. Effects of three heating rates on the critical thermal maximum of bluegill. In GIBBONS J.W. and SHARITZ R.R. eds, Thermal Ecology, U.S. Atomic Energy Commission Publ.,158-163.

COX D.K., COUTANT C.C., 1976. Acute cold-shock resistance of gizzard shad. In ESCH G.W. and MAC FARLANE R.W. eds, Thermal Ecology II, Technical Information Center Energy Research and Development Administration Publ.,159-161.

CURY P., ROY C., 1995. Exploration dans le non-linéaire des relations entre l'environnement et la dynamique des populations marines. In Méthodes d'études des systèmes halieutiques et aquacoles, $2^{\mathrm{e}}$ forum halieumétrique, ORSTOM éd., Nantes, 129-136, $277 \mathrm{p}$.

EATON J.G., SCHELLER R.M., 1996. Effects of climate warming on fish thermal habitat in streams of the United States. Limnol. Oceanogr., 41 (5), 1109-1115. 
ELIE P., ROCHARD E., 1994. Migration des civelles d'anguilles (Anguilla anguilla L.) dans les estuaires, modalités du phénomène et caractéristiques des individus. Bull. Fr. Pêche Piscic., 335, 81-98.

ESCH G.W., MAC FARLANE R.W., 1976. Thermal Ecology II. Proceedings of a Symposium. Technical Information Center, Energy Research and Development Administration ed., 404 p.

GIBBONS J.W., SHARITZ R.R., 1974. Thermal Ecology. Proceedings of a Symposium. United States Atomic Energy Commission ed., $670 \mathrm{p}$.

GIBBONS J.W., 1976. Thermal alteration and the enhancement of species populations. In ESCH G.W. and Mac FARLANE R.W. eds, Thermal Ecology II, Technical Information Center Energy Research and Development Administration Publ., 27-31.

HUTCHISON V.H., 1976. Factors influencing thermal tolerances of individual organisms. In ESCH G.W. and MAC FARLANE R.W. eds, Thermal Ecology II, Technical Information Center Energy Research and Development Administration Publ., 10-26.

KELEHER C.J., RAHEL F.J., 1996. Thermal limits to salmonid distributions in the Rocky Mountain region and potential habitat loss due to global warming : a Geographic Information System (GIS) approach. Trans. Amer. Fish. Soc., 125, 1-13.

JOBLING M., 1997. Temperature and growth : modulation of growth rate via temperature change. In WOOD C.M. and MAC DONALD eds (1997), Global warming : implications for freshwater and marine fish, Society for Experimental Biology, seminar series 61, Cambridge University Press, 225-253, $425 \mathrm{p}$.

LAPORTE E., 1853. Faune ichtyologique du département de la Gironde. In Actes de la Société Linnéenne de BordeauX, XIX (9), 158-224.

LARDEUX F., 1986. Biologie, écologie et dynamique de population de l'éperlan dans l'estuaire de la Loire. Thèse de Doctorat de 3ème cycle, Université de Bretagne Occidentale, Brest, $533 \mathrm{p}$.

LISCHKE H., LÖFFLER T.J., FISCHLIN A., 1997. Calculating temperature dependence over long time periods : derivation of methods. Ecological Modelling, 98, 105-122.

LYLE A.A., MAITLAND P.S., 1997. The spawning migration and conservation of smelt Osmerus eperlanus in the River Cree, Southwest Scotland. Biol. Conserv., 80, 303-311.

MAC ALLISTER D.E., 1963. A revision of the smelt family, Osmeridae. Department of Northern Affairs and National Ressources. Can. Bull., 191, 47 p.

MAC CARTHY I.D., HOULIHAN D.F., 1997. In WOOD C.M. and MAC DONALD D.G. eds (1997), Global warming : implications for freshwater and marine fish, Society for Experimental Biology, seminar series 61, Cambridge University Press, 51-77, $425 \mathrm{p}$

MAC FARLANE R.W., MOORE B.C., WILLIAMS S.E., 1976. Thermal tolerance of stream Cyprinid minnows. In ESCH G.W. and MAC FARLANE R.W. eds, Thermal Ecology II, Technical Information Center Energy Research and Development Administration Publ.,159-161.

MAITLAND P., 1990. Conservation of sparling and other fish in Scotland. Journal of the Edinburgh Natural History Society, 16-18.

MARTIN D., 1996. Global model of the dynamics and ecology of the population of allis shad (Alosa alosa) in the Gironde-Garonne-Dordogne watershed. International Council for the Exploration of the Sea, Anadromous and catadromous fish committee, CM 1996/M:11, $17 \mathrm{p}$.

MENDELSSOHN R., CURY P., 1987. Fluctuations of a fortnightly abundance index of the Ivoirian coastal pelagic species and associated environmental conditions. Can. J. Fish. Aquat. Sci., 44, 408-421.

MOYLE P.B., HERBOLD B., STEVENS D.E., MILLER L.W., 1992. Life history and status of delta smelt in the Sacramento-San Joaquin estuary, California. Trans. Amer. Fish. Soc., 121, 67-77. 
NELLBRING S., 1989. The ecology of smelts (Genus Osmerus) : a literature review. Nordic J. Freshwat. Res., 65, 116-145.

RAHEL F.J., KELEHER C.J., ANDERSON J.L., 1996. Potential loss and population fragmentation for cold water fish in the North Platte River Drainage of the Rocky Mountains : response to climate warming. Limnol. Oceanogr., 41 (5), 1116-1123.

RAMADE F., 1987. Eléments d'écologie : écologie fondamentale. Mac Graw Hill. ed., 343 p.

ROBITAILLE J.A., VIGNEAULT Y., 1990. L'éperlan arc-en-ciel (O. mordax) anadrome de l'estuaire du Saint-Laurent: Synthèse des connaissances et systèmatique de la restauration des habitats de fraie dans la rivière Boyer. Rapp. Manu. Canadien des Sc. Halieut. et Aquat., 2057, 56 p.

SCHENK H.J., 1996. Modeling the effects of temperature on growth and persistence of tree species : a critical review of tree population models. Ecological Modelling, 92, 1-32.

SEPULVEDA, 1994. Daily growth increments in the otoliths of European smelt Osmerus eperlanus larvae. Mar. Ecol. Prog. Ser., 108, 33-42.

THIEL R., SEPULVEDA A., KAFEMAN R., NELLEN W., 1995. Environmental factors as forces structuring the fish community of the Elbe estuary. J. Fish. Biol., 46, 47-69.

WOOD C.M. and MAC DONALD D.G. (eds), 1997. Global warming : implications for freshwater and marine fish. Society for Experimental Biology, seminar series 61. Cambridge University Press, $425 \mathrm{p}$. 